\title{
The future of plastic optical fiber
}

\author{
Yasuhiro Koike ${ }^{1,2 *}$ and Makoto Asai ${ }^{1,3}$ \\ Keio University, Japan
}

The number of services providing large-volume information content, such as high-definition movies, continues to increase rapidly. Single-mode glass optical fiber (SM GOF), which has been widely deployed in data trunk lines and pipelines connecting large cities and nations, has already become indispensable as an information transmission medium. However, SM GOF is mechanically weak and lacks sufficient bending ability. Moreover, as the core diameter is very small, just $10 \mu \mathrm{m}$, extremely precise techniques and expensive devices are required to connect fibers to signal receiving devices. It is therefore difficult to lay SM GOF for very short reach networks, such as local area networks in buildings. These difficulties are recognized as the problem of the 'last hundred meters' for optical fiber infrastructure. Plastic optical fiber (POF) has a relatively large-diameter core and is flexible enough to be laid down for network infrastructure in the last hundred meters. However, POF has two important weaknesses: it has significantly lower bandwidth than GOF, and its attenuation is far higher. Recent developments conquering both of these issues now mean that POF is regarded as the strongest candidate at present for optical data transmission over the last hundred meters. Here we review the status of POF development and research, particularly with respect to the application of 'photonics polymer'.

$\mathrm{T}$ he twentieth century, the so-called 'miraculous century of physics', has drastically changed our way of life. The advent of new physics, the relativity theory and the quantum theory, have dramatically enhanced technology, revolutionizing our vision of the universe. Today, people are in the midst of a new revolution, with our perspective of the world shifted by the concept of 'networks'. Twenty years after the development of low-attenuation single-mode (SM) silica glass optical fiber (GOF) in the 1970s [1], the United States launched the Information Superhighway project. The establishment of a global fiber-optic network has allowed the distribution of a wide range of media content, providing immediate access to new information. The world has become much smaller, eliminating physical constraints and endowing people with the ability to connect with anyone at anytime, living anywhere around the world. The internet has not only made our life more interesting and livable, but has created new industries, given rise to new cultures, and influenced politics. Indeed, it has changed our way of life.

However, there remains one final hurdle to achieving ubiquitous high-bandwidth connectivity: there is not yet a high-speed pipeline that connects users to the superhighway. In the 'last hundred meters' between the superhighway and users, especially in home and office local area networks (LANs), optical fibers must be bent at many points when laid down along walls. However, SM GOF is designed with a small core of just $5-10 \mu \mathrm{m}$ (Figure 1) so as to excite only one optical mode within the fiber, and is thus not capable of withstanding all the bends required in the last hundred meters. One solution that has been proposed is multi-mode (MM) GOF, which is designed with a larger core. However, the number of modes excited within MM GOF increases dramatically with enlargement of the core region, causing

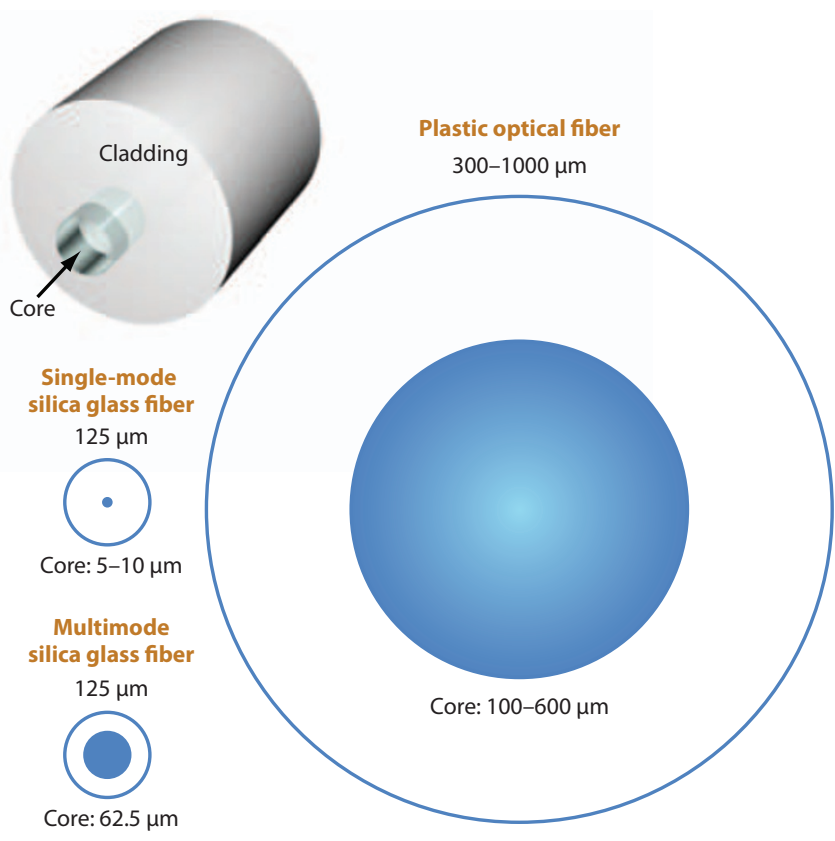

Figure 1. General construction of an optical fiber

the transmission rate to decrease accordingly due to an increase in the differences of the arrival times for each mode (modal dispersion). 

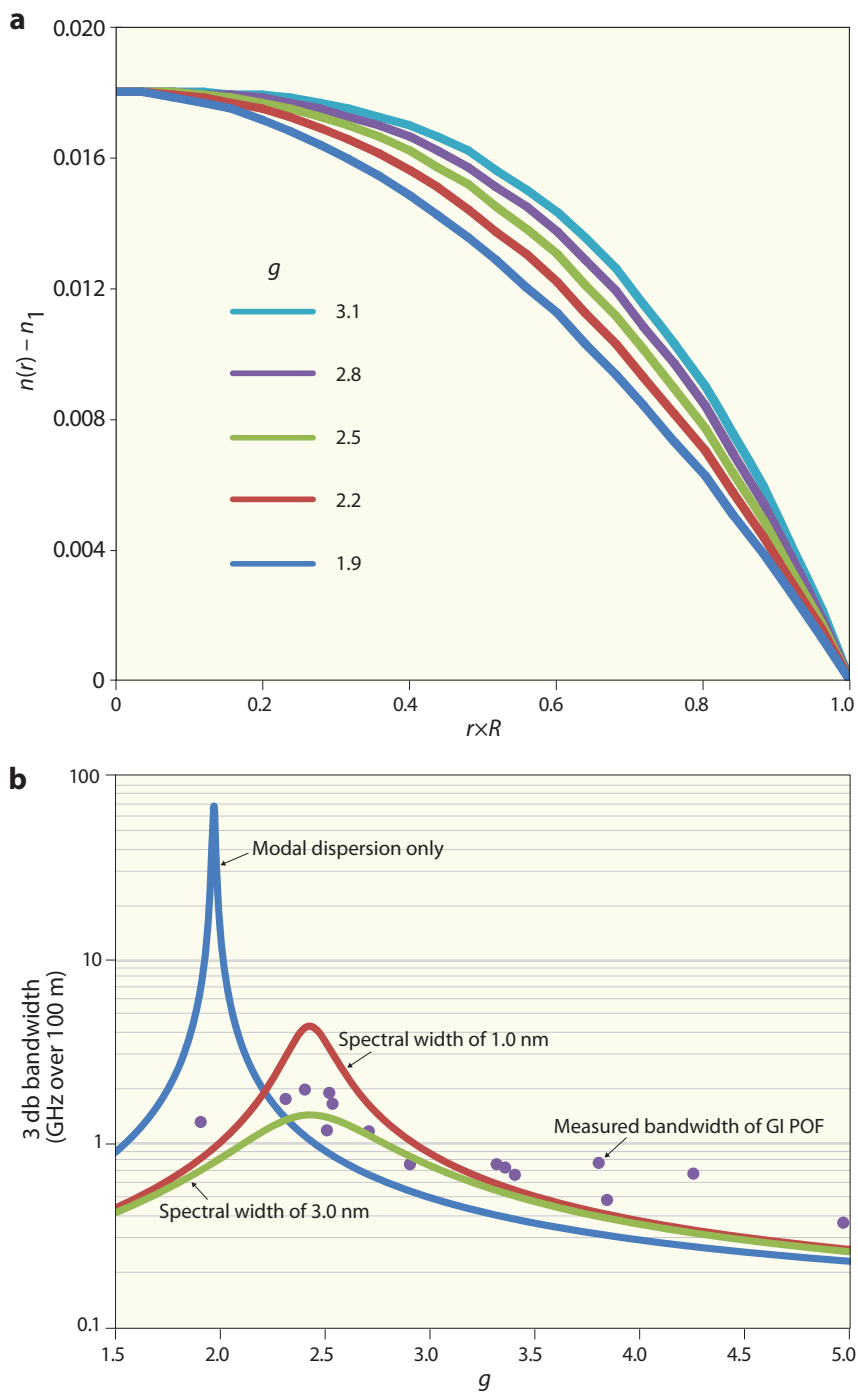

Figure 2. (a) General refractive index profiles of GI POF. (b) Relationship between index profile coefficient $g$ and $-3 \mathrm{~dB}$ bandwidth for $100 \mathrm{~m}$ of PMMA-based GI POF at $650 \mathrm{~nm}$ wavelength.

Nishizawa et al. developed graded-index (GI) MM GOF, which has a quadratic function-like graded index profile in the core region [2-4]. This fiber is designed based on the idea that excited modes will be refocused while propagating and modal dispersion will be reduced due to the lens-like function of the graded index profile in the core. Yet, at the initial stage of development, the attenuation of GI MM GOF was too high for practical use. Later, Kao et al. suggested that the attenuation could be reduced by eliminating impurities in the fiber [5]. Even then, an issue remained-the fiber diameter must be matched with extreme accuracy when connecting fibers. Although many connection devices are under intense investigation, they are generally expensive and are still not user friendly.

Currently, networks generally consist of SM GOF trunk lines and metal cables connecting the trunk lines to users. Light signals are converted to electric signals in this last node. This type of system does not require fiber alignment, and is thus easy to set up. However, the electrical signal intensity decreases with increasing signal frequency and transmission distance. Moreover, signals are easily affected by disturbances such as external electromagnetic interference and selfinduced electric fields. Hence, the maximum transmission rate for an electrical connection is no more than several hundred megabits per second (Mbps). Considering that the transmission rate of SM GOF is more than several hundred terabits per second, about 10,000 times faster than metal cables, users cannot at present benefit fully from the advantages of optical connections. Due to this last hundred meters issue, users are still unable to experience the high-speed performance of the SM GOF superhighway. The last hundred meters also cover more of the network than the trunk lines. When comparing networks to the human body, the trunk lines function like arteries and veins, while the last hundred meters are represented by the capillaries. It is estimated that this last hundred meters would correspond to $95 \%$ of all optical networks [6]. It is clear that the expected market is huge, and researchers all over the world are competing to find a solution to this problem.

Step index (SI) POF has been proposed as a candidate solution. SI POF has a larger core diameter, is more flexible than GOF, and is easy to handle [7]. Although the degradation of transmission rate caused by the increase in modal dispersion arising from the increase in core diameter has not been overcome, recent studies have shown that these disadvantages could be addressed indirectly by improving the transmission method, an approach that could realize an SI POF system capable of bit rates of over 1 Gbps [8-12]. On the other hand, studies of POF itself have also succeeded in overcoming this problem. The graded index (GI) POF has greatly reduced modal dispersion, allowing transmission at faster than 1 Gbps. The last hundred meters are thus finally being conquered through basic investigations of networks using POF. This review outlines the state of POF technology as it applies to the last hundred meters, and describes recent developments in POF focusing on the fundamentals of 'photonics polymer'.

\section{State of plastic optical fiber technology}

Plastic has gathered broad attention as an optimal candidate for a fiber material $[13,14]$. The first POF, invented in 1966 by Du Pont, had a step index profile. SI POF can be enlarged to about $980 \mu \mathrm{m}$ in diameter without losing its flexibility or ease of fiber alignment. SI POF is also advantageous in terms of mass production. Not only is SI POF inexpensive to fabricate, but it is also easy to mold and manufacture. Such fibers have high potential for realizing a complete high-speed optical network. The first commercialized SI POF was Eska, introduced by Mitsubishi Rayon in 1975, and Asahi Chemical and Toray also subsequently entered the market. Yet, the first fibers were not transparent enough to be used in the last hundred meters. With research on transparency by Kaino and colleagues, a complete high-speed optical network has become realistic [15]. Over the years, transparency has been enhanced to the theoretical limit [16-18]. Moreover, the thermal stability of POF, generally lower than that of silica, has been investigated from the three perspectives: the design of molecular structures having high thermal stability [19-23], the adoption of copolymers [24,25], and the use of cross-linked polymers [26-27]. By these basic investigations, the thermal stability of SI POF has been enhanced sufficiently for use in the last hundred meters. Its bandwidth has also been improved [29-35]. However, despite innumerous developments, improvement of the bandwidth of SI POF has its limitations. For transmission, it must excite several tens of thousands of modes, more modes than those found in MM GOF. The large core increases modal dispersion, drastically degrading its bandwidth to about several hundred megahertz over $100 \mathrm{~m}$.

However, many studies have been conducted on Gigabit Ethernet over SI POF, mainly in Europe. Although the transmission rate of SI POF is theoretically limited to several hundred megahertz over $100 \mathrm{~m}$, transmission rates exceeding $1 \mathrm{Gbps}$ have been achieved by adopting discrete multitone (DMT) modulation in networks using SI POF [8-12].

Many studies have been conducted to improve the bandwidth of POF. The modal dispersion can be reduced drastically by adopting an appropriate refractive index profile in the core region, as in MM GOF. Graded index (GI) POF was developed as one approach to reducing 
modal dispersion. General refractive index profiles, as shown in Figure 2(a), can generally be approximated by the following power law:

$$
\begin{aligned}
n(r) & =n_{1}\left[1-2 \Delta(r / R)^{g}\right]^{1 / 2}, & & 0 \leq r \leq R \\
& =n_{1}(1-2 \Delta)^{1 / 2}, & & R<r
\end{aligned}
$$

where $n(r)$ is the refractive index $(n)$ at radius $r$ of the fiber, $n_{1}$ is the refractive index at the core center, $n_{2}$ is the refractive index of the cladding, $R$ is the core diameter, $g$ is a refractive index profile coefficient, and $\Delta$ is the relative index difference defined by

$$
\Delta=\frac{n_{1}^{2}-n_{2}^{2}}{2 n_{1}^{2}} \cong \frac{n_{1}-n_{2}}{n_{1}}
$$

The refractive index profile coefficient defines the refractive index profile in GI POF. The bandwidth properties of GI POF with refractive index profiles expressed by equation (1) have been analyzed by the Wentzel-Kramers-Brillouin (WKB) method [36-38]. Generally, the bandwidth of optical fiber that excites many modes, such as POF, is dominantly influenced by modal dispersion. However, once modal dispersion is reduced, as in GI POF, the influence of material dispersion on bandwidth can no longer be ignored. Material dispersion is caused by the dependence of refractive index on the wavelength of light. According to analysis by the WKB method, modal dispersion $\sigma_{\text {inter, }}$ material dispersion $\sigma_{\text {intra }}$ and total dispersion $\sigma_{\text {total }}$ can be expressed as follows.

$$
\begin{aligned}
& \sigma_{\text {inter }}=\frac{L n \Delta}{2 c} \frac{g}{g+1}\left(\frac{g+2}{3 g+2}\right)^{1 / 2}\left[S_{1}^{2}+\frac{4 S_{1} S_{2} \Delta(g+1)}{2 g+1}+\frac{4 S_{2}^{2} \Delta^{2}(2 g+2)^{2}}{(5 g+2)(3 g+2)}\right]^{1 / 2} \\
& S_{1}=\frac{g-2-\varepsilon}{g+2}, S_{2}=\frac{3 g-2-2 \varepsilon}{2(g+2)}, \quad \varepsilon=\frac{-2 n_{1}}{n} \frac{\lambda}{\Delta} \frac{\mathrm{d} \Delta}{\mathrm{d} \lambda}, n=n_{1}-\lambda \frac{\mathrm{d} n_{1}}{\mathrm{~d} \lambda} \\
& \sigma_{\text {intra }}=\frac{L \sigma_{s}}{\lambda}\left[\left(-\lambda^{2} \frac{\mathrm{d}^{2} n_{1}}{\mathrm{~d} \lambda^{2}}\right)^{2}-2 \lambda^{2} \frac{\mathrm{d}^{2} n_{1}}{\mathrm{~d} \lambda^{2}}(n \Delta) S_{1}\left(\frac{2 g}{2 g+2}\right)+(n \Delta)^{2}\left(\frac{g-2-\varepsilon}{g+2}\right)^{2} \frac{2 g}{3 g+2}\right]^{1 / 2} \\
& \sigma_{\text {total }}=\sqrt{\sigma_{\text {inter }}^{2}+\sigma_{\text {intra }}^{2}}
\end{aligned}
$$

Here, the spectral width $\sigma_{s}$ is the wavelength dispersion of the input pulse, $c$ is the light speed, $\lambda$ is the wavelength of light and $L$ is the transmission distance. As $\sigma_{\text {intra }}$ is generally smaller than $\sigma_{\text {inter }}$, the bandwidth is mainly decided by $\sigma_{\text {inter }}$. However, when the bandwidth of the fiber is high enough, the influence of $\sigma_{\text {intra }}$ on the bandwidth cannot be ignored. Indeed, it is calculated that $\sigma_{\text {inter }}$ and $\sigma_{\text {total }}$ reach absolute minima at $g=2.1$ and $g=2.5$, respectively, in the case of GI POF fabricated from polymethylmethacrylate (PMMA) [39]. The relationship between the theoretical bandwidth and $g$ for PMMA-based GI POF at an operating wavelength of $650 \mathrm{~nm}$ is shown in Figure 2(b). The theoretical bandwidth was calculated using the wavelength dispersion of the light source at $\sigma_{s}=1.0$ and $3.0 \mathrm{~nm}$. As can be seen, the bandwidth of PMMA-based GI POF increases dramatically around the optimal refractive index profile coefficient, reaching a theoretical limit of about $3.0 \mathrm{GHz}$ over $100 \mathrm{~m}$. It can also be seen that the theoretical bandwidth is much lower when $g$ is not optimal. Therefore, it can be said that controlling the refractive index profile is critical for maximizing the bandwidth of GI POF.

Figure 3 shows the history of advancements in bit rates vs. distance for POF. From the 1990s up to the present day, the bandwidth of GI

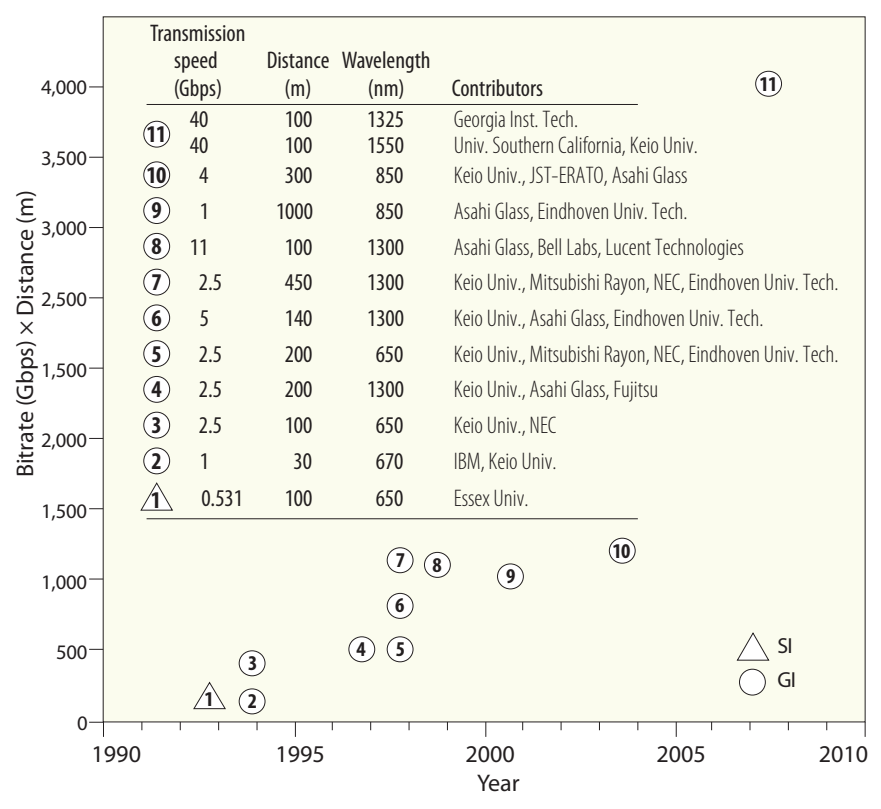

Figure 3. History of bit rates vs. distance advancements for POF.

POF has been steadily improving. Recently, a 100 m POF link capable of data transmission at $40 \mathrm{Gbps}$ was demonstrated.

\section{Development of GI POF}

Once it was realized that GI POF has considerably potential for resolving the problems of SI POF (the decrease in bandwidth caused by modal dispersion) without losing the flexibility of plastic, investigations into how GI POF can be produced were widely initiated. The approaches taken by these investigations can be broadly sorted into three major categories: (1) the fabrication of POF with a defined refractive index profile, (2) lowering the attenuation of GI POF for use in the last hundred meters, and (3) reducing material dispersion.

As a solution for how to fabricate GI POF, the interfacial-gel polymerization method [40] was proposed. In this method, monomers that have permeated through a polymer phase are polymerized sequentially starting from the polymer-monomer boundary, causing a gel effect. It was proposed that this method could be adapted to a copolymer system. The basic idea of this method is as follows.

First, a polymer tube is fabricated from a monomer with refractive index $n_{1}$. Monomers $M_{1}$ and $M_{2}$, with refractive indices $n_{1}$ and $n_{2}$ $\left(n_{1}<n_{2}\right)$, are then added to the tube. In this system, polymerization starts around the inner wall of the polymer tube (interfacialgel polymerization). When the reactivity ratio $r_{12}$ and $r_{21}$ between monomer $M_{1}$ and $M_{2}$ is $r_{12}>1$ and $r_{21}<1$, monomer $M_{1}$ is polymerized preferentially. As the polymerization front progresses toward the core, the promotion of the monomer $M_{2}$ increases. Therefore, the refractive index near the center of the core is higher than that near the cladding. GI POF was fabricated for the first time based on this idea [41]. MMAbenzyl acrylate (BzA), MMA-vinyl benzoate (VB) and MMA-vinyl phenyl acrylate (VPAc) GI POFs have been investigated, and their reported attenuations are about $200 \mathrm{~dB} / \mathrm{km}$ at $652 \mathrm{~nm}$ [40]. Such values are in the range of attenuation for commercial PMMA-based SI POF $(100-300 \mathrm{~dB} / \mathrm{km})$.

\section{Light scattering in polymers}

Despite the fact that GI POF could be fabricated, achieving complete copolymerization in a system containing monomers with vastly 
different reactivities remained a concern. The unpolymerized monomers and structural heterogeneities caused by incomplete polymerization could influence the scattering loss of the copolymer. The weight fraction of each monomer unit in the copolymer can be calculated by the Mayo-Lewis equation [42], as follows.

$$
y=\frac{\left(r_{1}-w\right) \chi^{2}+w \chi}{\left[w\left(r_{2}-1\right)+r_{1}-1\right] \chi^{2}-\left(2 r_{2} w-w-1\right) \chi+r_{2} w}
$$

Here, $y$ is the instantaneous weight fraction of the $M_{1}$ unit in the copolymer, and $r_{1}$ and $r_{2}$ are the reactivities of monomers $M_{1}$ and $M_{2}$. If $r_{2}$ is less than 0.5 , which is the case for the MMAVPAc monomer system, MMA monomer is consumed during polymerization and only homopolymers of $M_{2}$ are formed in the final stage of polymerization. Thus, when polymerization is completed, homopolymers of $M_{2}$ monomer exist in all cases of MMA-VPAc and MMA-VB [40]. In such a system, heterogeneities with larger refractive index are likely to generate light scattering loss, increasing the attenuation of the GI POF. However, the reported examples of GI POF did not have such large attenuation.

To investigate this disagreement, Debye's light scattering method [43] can be applied to quantify polymer heterogeneities [44]. In this method, it is assumed that light scattering loss in amorphous polymer glass can be expressed by

$$
V_{\mathrm{v}}=V_{\mathrm{V}_{1}^{\text {iso }}}+V_{\mathrm{V}_{2}^{\text {iso }}}+\frac{4}{3} H_{\mathrm{v}}
$$

where $V_{\mathrm{V}}$ is the polarized light scattering intensity, $V_{\mathrm{V}_{1}}$ iso denotes the isotropic background scattering independent of the scattering angle, $V_{\mathrm{V}_{2}}$ iso is the isotropic scattering that depends on the scattering angle due to large-sized heterogeneities and $H_{\mathrm{V}}$ is the depolarized light scattering intensity. Here, $V_{\mathrm{V}_{1}}$ iso is caused by density fluctuations induced by heat, and is formulated as Einstein's fluctuation theory [45].

$$
V_{V_{1}^{\text {iso }}}=\pi^{2}\left(n^{2}+2\right)^{2}\left(n^{2}-1\right)^{2} k_{\mathrm{B}} T \beta(T) /\left(9 \lambda_{0}^{4}\right)
$$

Here, $n$ is the refractive index, $k_{\mathrm{B}}$ is Boltzmann's constant, $T$ is absolute temperature, $\lambda_{0}$ is the wavelength of the light source in a

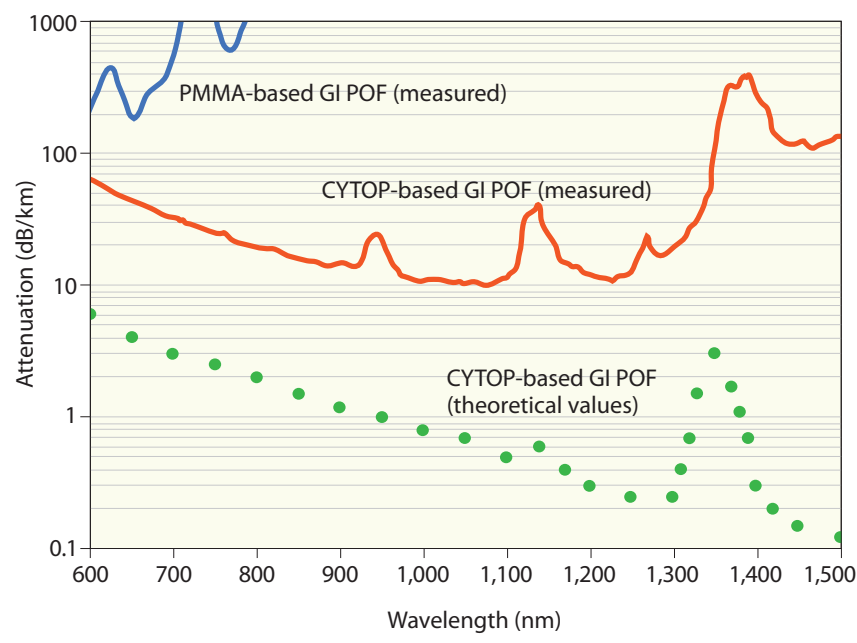

Figure 4. Comparison of attenuation spectra between PMMA and CYTOP GI POFs. Theoretical values for CYTOP determined by equation (18) are also shown. vacuum, and $\beta(T)$ is the isothermal compressibility. Debye's theory is then written as

$$
V_{V_{2}^{\text {iso }}}=\frac{4\left\langle\eta^{2}\right\rangle \pi^{3}}{\lambda_{0}^{4}} \int_{0}^{\infty} \frac{\sin (v s r)}{v s r} r^{2} \gamma(r) \mathrm{d} r
$$

with

$$
v=2 \pi n / \lambda_{0}, v=2 \sin (\theta / 2)
$$

Here, $\left\langle\eta^{2}>\right.$ denotes the mean-square average of the fluctuation of all dielectric constants, and $\gamma(r)$ refers to the correlation function defined by $\eta_{i} \eta_{j} /<\eta^{2}>$, where $\eta_{i}$ and $\eta_{j}$ are the fluctuations of dielectric constants at positions $i$ and $j$ separated by distance $r$. Debye et al. suggested that $\gamma(r)$ can be approximated by [44]

$$
\gamma(r)=\exp (-r / a)
$$

where $a$ is the correlation length, which expresses the magnitude of heterogeneities of the dielectric constant within the polymer. Substituting equation (12) into (10) and then integrating gives

$$
V_{V_{2}^{\text {iso }}}=\frac{8 \pi^{3}\left\langle\eta^{2}\right\rangle a^{3}}{\lambda_{0}^{4}\left(1+v^{2} s^{2} a^{2}\right)^{2}}
$$

The arrangement of equation (13) gives the Debye plot of $\left(V_{\mathrm{V}_{2}} \text { iso }\right)^{-1 / 2}$ versus $s^{2}$. The correlation length $a$ can be determined from the straight line of the plot by $a=\left(\lambda_{0} / 2 \pi\right)$ (slope/intercept $)^{1 / 2}$. Measurements of the correlation length have confirmed that in MMA- $M_{2}$ systems, $a$ is $350-600 \AA$ and $<\eta^{2}>$ is $6 \times 10^{-9}$ to $1.5 \times 10^{-8}$. This result contradicts the results of analysis by the Mayo-Lewis equation. If a blendlike polymer is formed in the MMA- $M_{2}$ systems, the calculated $<\eta^{2}>$ should be about $7 \times 10^{-3}$, which is approximately five orders of magnitude larger than the observed $\left\langle\eta^{2}\right\rangle$. From this result, it was determined that the two monomers are mixed at comparatively high density due to the specific nature of the polymerization reaction in the interfacial gel polymerization. Mutual diffusion of the two monomers could influence polymerization, since polymerization occurs locally in the interfacial gel polymerization method. Therefore, to form the required refractive index profile without increasing light

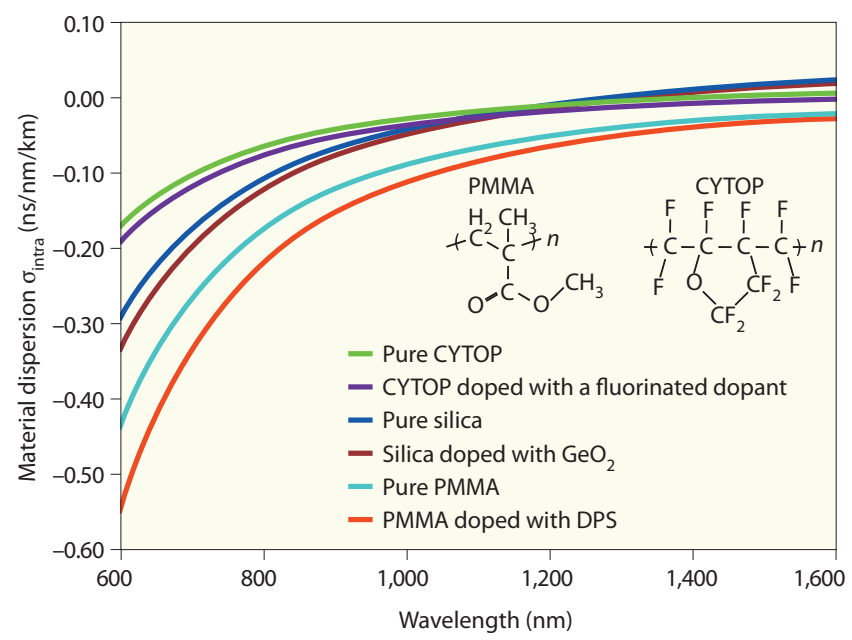

Figure 5. Comparison of material dispersion of polymer matrices for pure and doped CYTOP, silica and PMMA. 
scattering loss, the most suitable method is to diffuse inactive lowmolecular-weight molecules into the core center by the interfacial gel polymerization method. In this way, the fabrication method for GI POF with low attenuation was established [46].

\section{Fully fluorinated polymer}

The transparency and material dispersion problems have also been investigated extensively. PMMA, the most general material for POF, consists of many hydrogen atoms. These atoms have small atomic weight, and the peak overtone vibration absorption of the $\mathrm{C}-\mathrm{H}$ bond is found in the visible light region. Reducing the absorption loss caused by atomic vibration could lower attenuation dramatically.

The energy level of the absorption wavelength, showing the absorption of light at each wavelength, is expressed by $[47,48]$

$$
G(v)=v_{0}\left(v+\frac{1}{2}\right)-v_{0} \chi\left(v+\frac{1}{2}\right)^{2}
$$

Here, $v$ is the quantum number $(=0,1,2,3 \ldots), \chi$ is the anharmonic constant and $v_{0}$ is the fundamental frequency. The term on the righthand side is the harmonic vibration, and the second term is the anharmonic vibration. The overtone frequency is expressed as

$$
v_{v}=G(\mathrm{v})-G(0)=v_{0} \mathrm{v}-\chi v_{0} \mathrm{v}(\mathrm{v}+1)
$$

With the quantum number set as 1.0 :

$$
v_{0}=\frac{v_{1}}{1-2 \chi}
$$

Equation (16) can then be substituted into equation (15) to give

$$
v_{v}=\frac{v_{1} v-v_{1} \chi v(v+1)}{1-2 \chi} \quad(=2,3,4 \ldots)
$$

The overtone absorption frequencies can be calculated by equation (17) from $\chi$, which can be calculated from measurements of $v_{1}$ and $v_{2}$. Groh et al. estimated the attenuation $L$ by the overtone vibration absorptions of the $\mathrm{C}-\mathrm{H}$ and $\mathrm{C}-\mathrm{X}$ bonds by [48]

$$
L[\mathrm{~dB} / \mathrm{km}]=\left[3.2 \times 10^{8}\right] \frac{\rho N_{\mathrm{CX}}}{M}\left(\frac{E_{\mathrm{v}}^{\mathrm{CX}}}{E_{0}^{\mathrm{CH}}}\right)
$$

where $\rho$ is the polymer density, $M$ is the molecular weight of the monomer, $N_{\mathrm{CX}}$ is the number of C-X bonds per monomer, and $E v^{\mathrm{CX}} / E_{0}{ }^{\mathrm{CH}}$ is the vibration energy ratio of each bond to the fundamental frequency of the $\mathrm{C}-\mathrm{H}$ bond. It has thus been found that replacing $\mathrm{X}$ atoms with chlorine or fluorine is the most effective for reducing atomic vibration absorption. CYTOP, developed and trademarked by Asahi Glass, is a fully fluorinated polymer in which hydrogen atoms are replaced with fluorine atoms, and offers high thermal stability and very low material dispersion.

The wavelength dependence of attenuation in POF fabricated using CYTOP is shown in Figure 4, and the material dispersion of CYTOP and its chemical structure are shown in Figure 5. The absorption loss by atomic vibration is extremely low compared with other materials for GI POF, and the attenuation at $1.0 \mu \mathrm{m}$ is about $10 \mathrm{~dB} / \mathrm{km}$. As the theoretical limitation of attenuation of CYTOPbased GI POF is about $0.7 \mathrm{~dB} / \mathrm{km}$ at $1.0 \mu \mathrm{m}$ [40], it is expected that the attenuation could be lowered further by preventing contamination during the GI POF fabrication process. Moreover, as seen in Figure

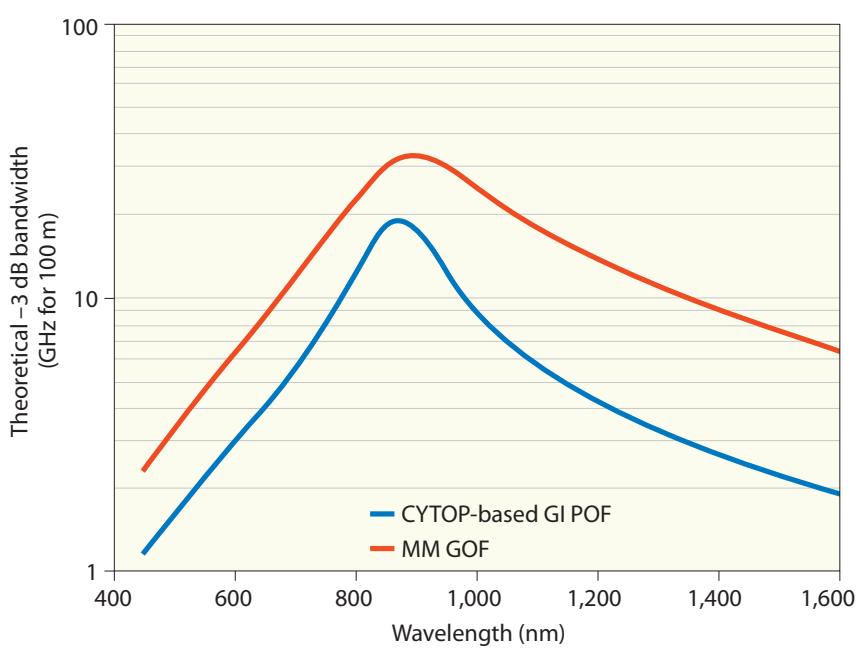

Figure 6. Dependence of theoretical $-3 \mathrm{~dB}$ bandwidth on wavelength in the CYTOPbased GI POF link compared with MM GOF $\left(\sigma_{s}=1.0\right)$. Bandwidth is calculated based on the assumption that each fiber has an optimal refractive index profile at $850 \mathrm{~nm}$.

5, CYTOP has low material dispersion compared to silica glass. The theoretical dependence of the bandwidth of CYTOP-based GI POF and MM GOF on wavelength is shown in Figure 6. CYTOP-based GI POF is predicted to have higher bandwidth than that of MM GOF. Indeed, in 1999, Asahi Glass and Bell Laboratories in the US reported successful experimental transmission at $11 \mathrm{Gbps}$ over $100 \mathrm{~m}$ using CYTOP-based GI POF [49]. This was an extremely significant result that proves POF can have higher bandwidth than MM GOF. CYTOP-based GI POF capable of $40 \mathrm{GHz}$ transmission over $100 \mathrm{~m}$ has recently been reported [50-52].

\section{Next stage of POF}

The review above is a historical overview of the fabrication of highbandwidth, low-attenuation GI POF for practical use. Currently, a novel mass production method for GI POF is being studied. Although the preform method adopting the interfacial gel polymerization method was essential in proving the usefulness of GI POF, the method remains too expensive to be adopted for mass production. Therefore, a new process for GI POF fabrication, called co-extrusion, has been investigated [53-55].

In this process, monomers doped with low-molecular-weight molecules and homogeneous monomers are polymerized as core and cladding polymers, respectively. The two materials are then melted in respective extrusion parts and compounded in a die to fabricate a POF that has a concentric circular core/cladding structure. At this point, the fiber does not have a graded refractive index distribution. By heating the fiber in the diffusion section, however, a radial concentration profile of low-weight molecules is formed as a result of molecular diffusion. Finally, the GI POF is obtained by winding it onto a take-up reel.

The co-extrusion process is very simple, and hence it is expected that GI POF can be manufactured continuously at relatively low cost. Yet there is still no method to control the refractive index profile. The diffusion mechanism of low-weight molecules within the polymer melt was investigated as a possible approach. If low-molecular-weight molecules only diffuse in the radial direction, the diffusion of these molecules can be expressed as

$$
\frac{\partial C}{\partial t}=\frac{1}{r} \frac{\partial}{\partial r}\left(D r \frac{\partial}{\partial r} C\right)
$$




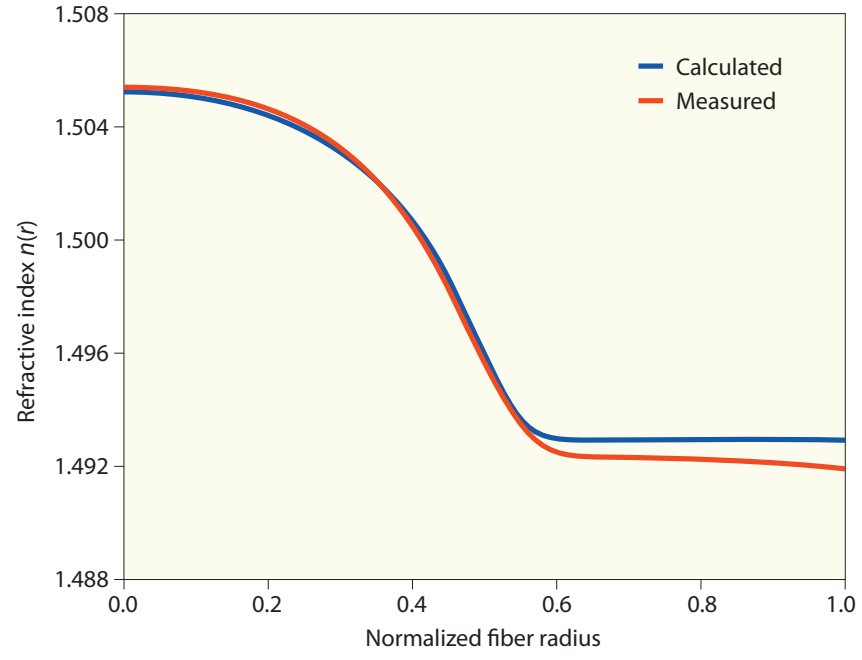

Figure 7. Refractive index profiles. Blue line is the refractive index profile calculated from equation (19) using the measured dependence of the mutual diffusion coefficient for a PMMA-DPS system. Red line is the measured refractive index profile for a PMMA-DPS-based GI POF fabricated by co-extrusion.

where $C$ is the concentration of low-weight molecules, $t$ is diffusion time, $r$ is distance from the fiber center and $D$ is the mutual diffusion coefficient of low-weight molecules. The concentration profile calculated from equation (19) has a gradual shape around the diffusion front, because $D$ is almost constant in a general system. Compared to the refractive index profile calculated from equation (1), that estimated by equation (19) has a larger tail around the diffusion front. This mismatch is caused by the degradation of bandwidth with increasing modal dispersion. Moreover, this tail part weakens the confinement of light, which is likely to deteriorate the bending capability of the fiber.

To investigate methods for minimizing this tail in the refractive index profile, we have measured the mutual diffusion coefficient $D$ of diphenyl sulfide (DPS) in molten PMMA [53]. The diffusion of a light-weight molecule can be modeled as follows. First, a onedimensional diffusion equation is converted to a nondimensional Botzmann equation:

$$
\frac{\mathrm{d} C(\eta)}{\mathrm{d} \eta}=-\frac{D}{2 \eta} \frac{\mathrm{d}^{2} C(\eta)}{\mathrm{d} \eta^{2}}
$$

where $\eta=x t^{1 / 2}$. Matano derived the concentration dependence of the diffusion coefficient using this equation [56]. Under appropriate boundary conditions, integrating the Boltzmann equation with respect to $\eta$ gives

$$
\begin{gathered}
-\frac{1}{2} \int_{C=0}^{C=C} \eta \mathrm{d} C=\left[D \frac{\mathrm{d} C}{\mathrm{~d} \eta}\right]_{C=0}^{C=C}=D t\left(\frac{\mathrm{d} C}{\mathrm{~d} x^{\prime}}\right)_{C=C} \\
\therefore D(C)=-\frac{1}{2 t}\left(\frac{\mathrm{d} x^{\prime}}{\mathrm{d} C}\right)_{C=C} \int_{C=0}^{C=C} x^{\prime} \mathrm{d} C
\end{gathered}
$$

where $x$ is called the Matano phase, and the coordinate system is defined with the origin at the initial core-cladding boundary. This 'BoltzmannMatano' method can be used to derive the concentration dependence of the diffusion coefficient. This method was applied to the concentration

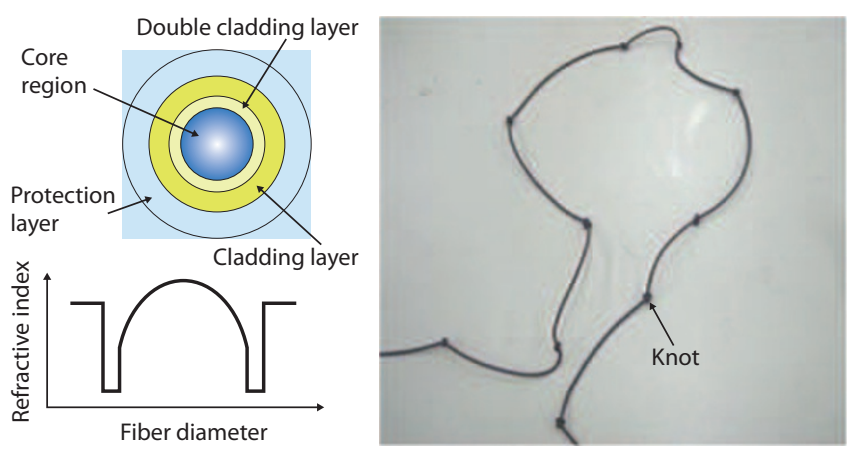

Figure 8. (Left) Structure and refractive index profile of double-cladding GI POF. (Right) Photograph of double-cladding GI POF with 10 knots (each knot has a bend radius of $3.0 \mathrm{~mm}$ ).

profile measured in a one-dimensional diffusion experiment. It was thus found that $D$ is exponentially dependent on the DPS concentration [54]. Therefore, the tail part of the refractive index profile can be made smaller by reducing the diffusivity of low-molecular-weight molecules around the diffusion front. This confirmed that there was a possibility of realizing high-bandwidth GI POF by the co-extrusion process. Figure 7 shows the refractive index profile of PMMA-DPS-based GI POF fabricated by this process. As predicted, there is no tail part in the diffusion front, and its mutual diffusion coefficient agrees well with that calculated by numerical integration of equation (19). In other words, it was confirmed both experimentally and theoretically, for the first time, that high-bandwidth GI POF could be fabricated by the co-extrusion process. Recently, investigations have also been made into how to accurately and efficiently control the refractive index profile in order to fabricate high-bandwidth GI POF more reliably [57].

The co-extrusion process also has the advantage that GI POF with novel structures, which are often difficult to realize by conventional methods, can be fabricated easily. For example, a novel GI POF called a 'double cladding' fiber was recently developed to reduce bending loss, an essential factor to consider when using optical fiber, especially POF, in home networks. Bending loss in this case is reduced by increasing the difference between the refractive indices of the core and cladding. The double cladding GI POF has two cladding layers, each with lower refractive index than that of conventional cladding materials (Figure 8). It has been reported that the data transmission rate required for the high-definition multimedia interface (HDMI) standard (10 Gbps) has been achieved in a system using the double cladding GI POF, even when knotted ten times (Figure 8). Novel POFs with other structures, such as multiple GI cores, are being investigated vigorously all over the world [58].

The latest research on POF has led to easier-to-handle and higherbandwidth fiber compared to conventional silica optical fiber, and POF is now ready for use in pipelines connecting to the superhighway. At this moment, the data rate of 10 Gbps needed for HDMI full high-vision television in the home can no longer even be satisfied by traditional copper cable.

\section{Summary}

This article reviewed the status of POF development and the latest research achievements focusing on GI POF as a promising candidate for the next generation of optical fiber. Basic research on POF technology has led to the creation of the novel academic field of 'photonics polymer'. There is a possibility that this new field will produce the technologies that will be used in all optical networks in the near future. The technology forms the basis for the 'fiber-to-the-display' concept, in which all optical networks will be directly connected by GI POF to high-definition displays in homes and offices, allowing real-time face-to- 
face communications with clear motion picture. This concept is not a continuation of the existing keyboard-oriented information technology, but will instead help to bring back face-to-face communications. We hope that POF will shed light upon the world in the future.

\section{References}

[1] D. B. Keck, P. C. Schultz, F. Zimar, Appl. Phys. Lett. 21, 215 (1972).

[2] A. G. Fox, T. Li, Bell System Tech. J. 40, 453 (1961).

[3] S. Kawakami, J. Nishizawa, J. Applied Phys. 38, 4807 (1967).

[4] S. Kawakami, J. Nishizawa, IEEE Transactions on Microwave Theory and Techniques MIT-16, 814 (1968)

[5] K. C. Kao, G. A. Hockham, Proc. IEE 113(7), 1151 (1966).

[6] W. Daum, J. Krauser, P. E. Zamzow, O. Ziemann, Polymer Optical Fibers for Data Communication, Springer, USA (2001).

[7] H. Yuuki, K. Asada, Optronics 220, 222 (1995).

[8] S. Randel et al., Proc. Eur. Conf. Optical Commun. Th4.4.1. (2006)

[9] F. Breyer, S. C. J. Lee, S. Randel, N. Hanik, Proc. Eur. Conf. Optical Commun. 9.6.6 (2007).

[10] J. Lee, F. Breyer, S. Randel, H. van den Boom, T. Koonen, Proc. Int. POF Conf. 17 (2008).

[11] O. Ziemann et al., Proc. Int. POF Conf. 17 (2008).

[12] F. Breyer, S. C. J. Lee, S. Randel, N. Hanik, Proc. ECOC We.2.A.3 (2008).

[13] R. J. S. Bates, IEEE Photon. Technol. Lett. 4, 1154 (1992).

[14] S. D. Walker, R. J. S. Bates, Proc. Int. POF Conf. 8 (1993).

[15] T. Kaino, M. Fujiki, S. Oikawa, S. Nara, Appl. Opt. 20, 2886 (1981).

[16] M. H. Schleinitz, Proc. Int. Wire \& Cable Smp. 26, 352 (1977).

[17] T. Kaino et al., Appl. Phys. Lett. 42, 567 (1983).

[18] T. Kaino et al., Appl. Phys. Lett. 48, 757 (1986).

[19] T. Yamashita, K. Lamada, Jpn J. Appl. Phys. 32, 2681 (1993).

[20] T. Yamashita, Jpn J. Appl. Phys. 33, 4025 (1994).

[21] S. Irie, M. Nishiguchi, Proc. Int. POF Conf. 4 (1994).

[22] A. Start Wood, Proc. Modern Plastics International 36 (1992).

[23] S. Teshima, H. Munekuni, S. Katsuta, Proc. Int. POF Conf. 44 (1992).

[24] S. Taneichi et al., Proc. Int. POF Conf. 106 (1994).

[25] S. Taneichi et al., Proc. Int. POF Conf. 111 (1995).

[26] T. Abe, H. Asano, K. Okino, N. Taketani, T. Sasayama, SAE Technical Paper Series,
910875 (1991).

[27] Y. Takezawa, S. Tanno, N. Taketani, S. Ohara, H. Asano, J. Appl. Polym. Sci. 42, 2811 (1991).

[28] Y. Takezawa, S. Tanno, N. Taketani, S. Ohara, H. Asano, J. Appl. Polym. Sci. 42,3195 (1991)

[29] M. Yaseen et al., Proc. OFC/IOOC ThC 5 (1993).

[30] S. Takahashi et al., Electron. Lett. 27, 217 (1991).

[31] K. Nakamura et al., Proc. ATM Forum PHY SWG AF 0919 (1995).

[32] R. J. S. Bates, S. D. Walker, Electron. Lett. 28, 996 (1992).

[33] S. Yamazaki et al., Proc. ECOC 337 (1995).

[34] H. Munekuni, S. Katsuta, S. Teshima, Proc. Int. POF Conf. 148 (1994).

[35] D. Gloge, Appl. Opt. 11, 2506 (1972).

[36] P. K. Tien, J. P. Gordon, J. R. Whinnery, Proc. IEEE 53, 129 (1965).

[37] J. P. Gordon, Bell System Tech. J. 45, 313 (1966).

[38] R. Olshansky, D. B. Keck, Appl. Opt. 15, 483 (1976).

[39] Y. Koike, T. Ishigure, J. Lightw. Technol. 24, 4541 (2006).

[40] Y. Koike, Polymer 32, 1737 (1991).

[41] Y. Ohtsuka, Y. Hatanaka, Appl. Phys. Lett. 29, 735 (1976).

[42] V. E. Meyer, G. G. Lowry, J. Polym. Sci. 3, 2843 (1965).

[43] P. Debye, J. Appl. Phys. 17, 392 (1946).

[44] Y. Koike, S. Matsuoka, H. E. Bair, Macromol. 25, 4807 (1992).

[45] A. Einstein, Ann. Phys. 33, 1275 (1910).

[46] Y. Koike, US Patent 5541 247, JP Patent 3332 922, EU Patent 0566 744, KR Patent 170 358, CA Patent 2098604 (originally filed in 1991).

[47] W. Groh, Makromol. Chem. 189, 2861 (1988).

[48] W. Groh, A. Zimmerman, Macromol. 24, 6660 (1991).

[49] G. Giaretta, M. Wegmueller, R. V. Yelamarty, Proc. Optical Fiber Commun. Conf. PD-14 (1999).

[50] J. Yu, M.-F. Huang, P. N. Ji, T. Wang, Proc. OFC (2008).

[51] A. Polley, S. E. Ralph, Proc. OFC OWB2 (2008).

[52] S. R. Nuccio et al., Proc. ECOC (2008).

[53] M. Asai, R. Hirose, A. Kondo, Y. Koike, J. Lightw. Technol. 25, 3062 (2007).

[54] I.-S. Sohn, C.-W. Park, Ind. Eng. Chem. Res. 40, 3740 (2001).

[55] I.-S. Sohn, C.-W. Park, Ind. Eng. Chem. Res., 41, 2418 (2002).

[56] C. Matano, Jpn J. Phys. 8, 109 (1933).

[57] M. Asai, K. Nehashi, Y. Koike, J. Lightw. Technol. 26, 2909 (2008).

[58] C. Tanaka, K. Kurashima, M. Naritomi, A. Kondo, Y. Koike, Proc. OFC/IOOC OWG 6 (2008).

\section{Author profiles}

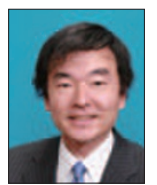

\section{Yasuhiro Koike}

Yasuhiro Koike was born in Tokyo, Japan, in 1954. He received his BS degree from Keio University in 1977, and earned his MS and PhD degrees in applied chemistry at the Graduate School of Engineering of Keio University, Japan, in 1979 and 1982, respectively. He was a visiting researcher with AT\&T Bell Laboratories from 1989 to 1990. Since 1997, he has held the post of professor at Keio University, where he developed high-bandwidth graded-index polymer optical fiber. Since 2005, he has also held the concurrent position of research director of the Exploratory Research for Advanced Technology and Solution-Oriented Research for Science and Technology (ERATO-SORST) project of the Japan Science and Technology Agency (JST).

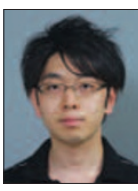

\section{Makoto Asai}

Makoto Asai was born in Ibaraki, Japan, in 1981. He earned his BS, MS and PhD degrees at Keio University, Japan, in 2005, 2006 and 2008, respectively. He now serves concurrently as a research fellow of the Japan Society for the Promotion of Science and a visiting researcher at Keio University. His current studies focus on glass transition and diffusion phenomena in polymers. In 2006-2007, he worked as a research assistant for the 21st Century Center of Excellence, and from 2007 has worked as a research assistant with the Exploratory Research for Advanced Technology and Solution-Oriented Research for Science and Technology (ERATO-SORST) project of the Japan Science and Technology Agency (JST). 